treatment, or as to the action of any remedy in the Pharmacopoia; and neither the College of Physicians, nor any of our medical societies, has made the slightest effort to obtain such a record. Here, then, is an opportunity for the Harveian Society to step in, and do a real service to the profession. The committee might test at the bedside the accuracy of the statements made by individuals respecting the so-called natural course of disease, and endeavour to ascertain, and impartially record, the amount of assistance which may fairly be expected from the vis medicatrix natura. They might investigate the action of certain drugs, the doses in which they might be safely administered, and the conditions which appear to modify their action; they might note the action of given remedies, in definite doses, or in certain combinations, in modifying the course of certain disorders, and might verify the various statements which have been made respecting their remedial powers. Observations of this kind, even though limited to a few drugs and very few diseases, if carefully made and briefly recorded, would serve as a model for furthe investigations, and'as a basis for a work, which within a few years, would be considered as needful to the medical practitioner as the Pharmacopaia is at the present time.

In another manner, too, I believe, a therapeutical committee would prove most useful. They would collect information about, and bring to the test of bedside experience, the reputed action of remedies not commonly employed, and the application of well known remedies, or unusual doses of remedies, to the relief of disorders for which they are not commonly prescribed. There are numerous practitioners throughout the country, who have acquired a great local reputation for the cure of certain forms of complaint; and it has come to my knowledge in several instances, that their reputation has been well deserved, and has been gained by the employment of remedies not commonly in use for the cure of those disorders, or by the administration of combinations or doses of medicines which are not usually prescribed. This sort of information rarely finds its way into print, or, if it does, attracts little attention. It is handed down from father to son, or from master to apprentice, and so, perhaps, ultimately becomes known to the profession; but much of it is necessarily lost, and will be so to a far greater degree now that the system of preliminary education is being changed. This information the committee might readily collect and submit to the proof of bedside experience.

So again, with regard to the doses of medicines and to their administration for the relief of certain disorders: no person who has not made inquiries on the subject can have an idea of the wide discrepancy which exists, between the practice of different medical men, in relation to the doses in which remedies are administered. Ignorance respecting drugs and their actions is so general, that in the absence of any authoritative statement on the subject, the vaguest and most groundless fears are entertained respecting their action; one physician, for instance, is in the habit of prescribing forty-minim or drachm doses of tincture of cantharides, but never ventures on more than ten or fifteen-minim doses of tincture of digitalis; whilst another, who habitually orders thirty or forty minims of tincture of digitalis, and in certain instances gives drachm or drachm-and-a-half doses, is not aware that tincture of cantharides is ever given in doses exceeding twenty minims, and himself has never prescribed more than ten or fifteen minims. And so with regard to numberless other remedies. We have no public or authoritative statement respecting the doses in which any medicine can be given with impunity, nor respecting the disorders in which it proves useful; and therefore most practitioners do as they have been taught, and oftentimes have no knowledge as to its administration in any other doses. But if the smaller doses suffice to produce the characteristic effect of the medicine, it can scarcely be doubted that the larger doses must be pernicious; whereas, on the contrary, if the larger doses are really needed, the smaller doses must obviously be inefficient. In either case the patient suffers: in the one, from being unnecessarily drugged; in the other, from losing valuable time, at the risk, perhaps, of his life, in taking doses which are inadequate to relieve his suffering. This extreme contrariety of practice, which is a standing reproach to our profession, might be removed through the investigation of a committee, and the establishment of something like a definite knowledge respecting the doses of medicines. The Harveian Society would thus obtain the honour of having inaugurated an inquiry which would not have been unworthy of the Royal College of Physicians.

Misconduct of Alleged Medical Students. - At the Middlesex Sessions, lately, George Richmond, John Hind, Robert Foss, and Alfred Swinborne, medical students, pleaded "Guilty" to assaulting Police-constable Cave, $35 \mathrm{C}$, and Allwood, $186 \mathrm{C}$, in the execution of their duty. Mr. Cooper prosecuted; Mr. F. H. Lewis defended. The Assistant-Judge said he should require time to consider what course ought to be taken, and postponed sentence until next sessions.

\section{RESEARCHES ON THE ACTION OF THE HEART.*}

By GEORGE PATON, M.D., Bowmanville, Canada West.

IF the continued action and varied degrees of rapidity with which the ventricles dilate cannot be accounted for by mere muscular relaxation considered by itself, we might argue that, as the dilatation of the ventricles takes place at the same time that the auricles contract and project their blood into the ventricles, it must either depend on some inherent power possessed by the ventricles, or it must be produced by the impetus imparted by the blood from the auricles to the relaxing parietes of the ventricles, causing them more rapidly to expand and reach the point of distension, when contraction is produced.

The great difficulty which has presented itself to physiologists in investigating this subject is that, during the vigorous action of the heart, the diastole of the ventricles appears to take place with an energy that cannot be accounted for by the mere relaxation of the fibres-by their returning to a state of rest; whilst at the same time the auricles are considered the reservoirs or receptacles into which the blood enters on its return from the system, and before it is projected into the dilating ventricles, it being believed that the auricles contract but slightly. Hence Pechlin, Perrault, Bichat, Laennec, Carson, Cruveilhier, and several other physiologists who have examined the action of the heart in living animals whilst respiration was well maintained, observing the vigour with which the diastole is performed, considered that it depends on an actively dilating power possessed by the ventricles; and it undoubtedly bears the strongest resemblance to a movement of this character. In a paper which we published in the Dublin Quarterly Medical Fournal for August 1866 , we stated that the force with which the ventricles dilate affords every indication of a vital active movement; and that it must either depend on some inherent power possessed by the ventricles, or be produced by the force with which the blood is projected by the auricles into the ventricles, stimulating the parietes, and causing their fibres to extend and complete the dilatation. And, on more fully investigating this subject, we incline to the latter opinion. The whole of the arricles, it is to be observed, contract at this period, and not the mere appendices; and they contract with a force that exerts an influence on th dilating parietes of the ventricles. We have frequently seen, as formerly stated, the auricles contract with a power that caused the blood to open up the walls of the ventricles, the fibres distinctly relaxing till distension was produced and contraction commenced; and Dr. John Reid states that the systole of the auricles is performed with great rapidity when the action of the heart is still vigorous, and appears to be effected by the simultaneous contraction of all its fibres. According to the observations of Harvey, Lower, Senac, Haller, and others, the contractions of the auricles are performed with considerable force. In the experiments of Dr. Hope, Mr. Carlisle, M. Bouillaud, and the Dublin Committee for Investigating the Cause of the Sounds of the Heart, the contraction of the auricles appeared to be comparatively trifling, and was most apparent in the appendices. From my own experiments upon rabbits and dogs, I am convinced that the auricles contract considerably more when the movements of the heart are proceeding in a natural manner, than some of these last experiments would lead us to believe; and that this contraction is not confined to the appendix, but extends over the whole auricle. That, however, none of the muscular fibres of the auricles are passive, but exert a force proportionate to their strength, we have evidence both from experiment and from the effects of disease.

And, if we examine the action of the heart when it has been exposed in large warm-blooded animals, we perceive the auricles contract with vigour; and, as they project the blood into the ventricles, the ventricles expand with a force sufficient to separate the fingers that seize them. We denuded the heart of a calf after the animal had been rendered insensible by a blow upon the head, and artificial respiration was maintained. The heart beat at 62 pulsations per minute; and it was surprising with what energy the action was maintained. The contraction of the auricles was synchronous with the diastole of the ventricles, and the whole of the auricles contracted, and not the mere appendices. The diastole of the ventricles was performed with a force that opened up the hand grasping the parietes; and it appeared to be produced by the impetus of the blood from the auricles; for the parietes of the ventricles could be distinctly seen and felt gradually to expand, and with a proportionate vigour, as the blood from the auricle shot to the apex. The ventricles first dilated, and then contracted. During the diastole, the anterior parietes became slightly protuberant: during the systole, they

* Read before the Physiological Section at the Annual Meeting of the British Medical Association in Dublin, August 1867. [Concluded from p. I64.] 
appeared as if depressed, and the apex was tilted up; but the action was maintained with great quickness and regularity.

The whole question resolves itself into very narrow limits. Dr. Hope, and the physiologists who adopt his views, admit that the auricles are the primum mobile or cause of the movement of the ventricles; that, in proportion to the vigour and rapidity with which the auricles contract and distend the ventricles, their contractile energy is increased, causing them to contract with greater power. On this principle, a force is exerted in producing distension and immediate contraction of the ventricles, for these are inseparably connected; and the interval is occupied by the mere relaxation of the muscular fibres. But, if it be admitted as a fact-which, we conceive, rests on incontrovertible data-that, during the vigorous action of the heart, the contraction of the auricles is synchronous with the commencement of the diastole of the ventricles, an active power is exerted both during the diastole of the ventricles and the systole; because, if the auricles have the power of increasing the contractile energy of the ventricles by the force which they exert in bringing them to a state of distension, it is evident that, in producing this effect, the auricles must increase the relaxation of the ventricles. Suppose, for instance, that the heart is beating at the rate of 50 pulsations per minute, and that it is gradually increased to 60,70 , or 80 in the same period, at every successive movement the auricles contract with greater energy, bringing the ventricles more rapidly to a state of distension, thereby increasing the power and energy with which they contract. But, as the force with which the auricles act extends through the whole period of the diastole of the ventricles, it must increase the rapidity with which the ventricles dilate whilst it is causing them to contract with greater energy ; that is to say, the one effect must be produced before the other, or congestion would quickly come and arrest the action of the heart. Thus we perceive how the action of the heart is increased by the systole of the auricles being synchronous with the diastole of the ventricles, the two movements proceeding in a regular and uninterrupted manner, and in quick and rapid succession. And not only is this the case, but, when the auricles contract with still greater rapidity, the diastole of the ventricles is performed with greater energy, and the systole with similar velocity. Hence, when the circulation is quick and rapid, the whole of the diastole of the ventricles, as well as the systole, is regulated by the auricies or is directly under their influence.

It is always dangerous to compare the physiological laws which reguate the functions of the animal economy with the physical laws that regulate the movements of inorganic matter, as the principle of vitality is connected with the one, and excluded from the other. Perhaps, however, we may be permitted, for the sake of illustration, to observe that, in the vertical steam-engine, the piston which is attached to the beam that moves the whole machinery is raised by a current of steam admitted into the cylinder; but it is not allowed to fall by the power of gravitation or atmospheric pressure. The moment it reaches a certain height, another current of steam is directed against it, to depress it; so that an active power is employed, both in raising and in depressing the piston, which greatly increases its velocity, sometimes attaining 200 strokes and upwards in a minute.

And, when the action of the heart is slow, the dilatation of the ventricles is the mere relaxation of their fibres after contraction; but, when the action is quick and rapid, the blood projected by the auricles increases the diastole of the ventricles, as well as produces the systole; so that the auricle exerts a direct power upon the ventricle, both when it dilates and when it contracts.

There is another aspect in which this subject may be viewed; and that is, by a sort of numerical calculation of the different periods in which the diastole and the systole are performed, and how this accords with the increased rapidity of the circulation of the blood. Suppose that the heart pulsates at the rate of 60 times per minute, equal to one pulsation in a second: the first stage of the diastole occupies onefourth of a second, and is described as an instantaneous expansive movement, by which the ventricles return to the same state with regard to form, size, and position as during the previous pause; then the pause occurs in one-eighth of a second; and in the other one-eighth, the auricles contract and distend the ventricles with blood, completing the second stage of the diastole; whilst the ventricles contract in the remaining half-second. But suppose that the action of the heart increases to 90 pulsations per minute, or one pulsation in two-thirds of a second: the first stage of the diastole occurs in one-sixth of a second, which is contrary to the doctrine of an instantaneous expansive movement; and the pause occupies one-twelfth of a second, and in one-twelfth of a second the auricles contract and distend the ventricles; whilst the ventricles contract in the remaining one-third of a second. But when the action of the heart is rapid, as at this rate of speed, it is generally admitted by physiologists that the whole of the auricles contract, and not the mere appendices; and that they contract with force. But it is difficult to perceive how the second stage of the diastole can be completed in one-twelfth of a second, or that twelve of these movements would occur in one second of time; and this will be still more the case when the action of the heart increases to I20 pulsations per minute, for then the second stage of the diastole will be completed in one-sixteenth of a second, or sixteen of these movements could be performed in one second of time.

But, according to the views which we maintain, the pause occurs only when the action of the heart is slow, and disappears when it is rapid. When the ventricle pulsates at the rate of $\mathbf{1 2}$ or 20 times a minute, the pause occurs, and is long, the blood being imperfectly arterialised; a due stimulus is not imparted to the heart; and all the functions of the body are languid. When the action of the heart increases to 30 or 40 pulsations per minute, the pause is shorter, and the movements are more energetic ; but, when the pulsations increase to 50 or 60 per minute, respiration being well maintained, the pause entirely disappears, and the diastole is synchronous with the contraction of the auricles, and occurs in half a second or thereabouts, and the systole in half a second. When the action increases to 90 pulsations per minute, the diastole occupies one-third of a second or thereabouts, and the systole one-third ; and the movements proceed in this manner, the diastole being quickly performed, and succeeded by the systole; and, when the action of the heart increases to 180 pulsations per minute, as in the heron and some other animals whose blood is highly arterialised, three pulsations occur in one second of time, allowing one-sixth for the diastole, and one-sixth for the systole.

We may now shortly inquire how the diastole of the auricles is produced. The auricles, it is well known, are never empty of blood, a portion remaining after contraction dilating their walls. The parietes of the auricles are very thin, and, during relaxation, very distensible or easily dilated. The auricles possess no valves behind them; but a free communication exists between them and the extremities of the veins, which are possessed of the power of contracting and dilating with the fibres of the heart. In cold-blooded animals, when the action of the heart is vigorous, the venous trunks or extremity of the veins contract first, then the auricles; after that the ventricle, and then the bulb of the aorta; there being, as Müller has remarked, nearly the same interval between them. But, when the circulation is slower, we have observed the blood in the vein, on its return from the body, continue to dilate the auricles and cause them to contract, independently of contraction of the venous trunks, showing that the blood returns along the veins with a power sufficient to distend the auricles and sustain the action of the heart. And this also appeared to take place without a vis a fronte; for, in cases where respiration had ceased and the ventricles were dilated and filled with blood, the blood, on its return from the extremities; continued to dilate the auricles and over-distend their parietes.

During these experiments, the action of the heart was always increased by the animals continuing to respire for a time. The blood, becoming more highly arterialised, appeared to impart a greater stimulus to the internal parietes of the auricles and ventricles; and it is well known, also, that exercise or increased exertion quickens the circulation in the veins, and causes the blood to return with greater vigour to the heart.

But it appears that, independently of this principle, the auricles are capable of being excited by a stimulus conveyed through the medium of the cerebral or sympathetic nerves to the heart. We have frequently seen in the turtle, and also in other animals whose heart has been denuded when the animal was remaining calm and quiescent, the action of the heart proceeding with much regularity, that suddenly, as if by a strong impulse, the auricles commenced to contract with great energy, and transmit the blood rapidly into the ventricle, increasing its action and these movements would continue for a time, and then subside, the calm and uniform action of the heart being restored.

During our experiments on large warm-blooded animals, as the calf, whose thorax had been laid open and the pericardium slit up, artificial respiration being maintained, we have found that the heart beat at 60 per minute and upwards; and, on carefully examining its action, both by placing our fingers against its parietes and observing the movements with the eye, we perceived that the blood passed rapidly along the veins to the heart; and, as it distended the parietes of the auricle, a strong impulse was communicated to the fingers that pressed it at the moment.

We have not, in the course of these observations, taken into account a partial vacuum occurring in the thorax during the dilatation of the heart and the act of inspiration, considered to increase the motion of the blood towards the heart; because in the fish, the frog, and even in the bird, this principle does not obtain during inspiration; and yet, in all these classes, the circulation is maintained with vigour. Nor does it occur in warm-blooded animals when the thorax has been laid open and the pericardium slit up, to enable us to observe the action of the heart. And, if a partial vacuum obtain during the diastole of the auricles, it does not affect or invalidate the view which we maintain, but rather 
supports it. What we are endeavouring to show is, that the blood, on its return from the body, enters the parietes of the auricles with a force which is capable of increasing the relaxing power of the auricles, or of bringing them rapidly to a state of distension, causing the auricles to contract with greater energy.

It must be admitted, however, that, when a partial vacuum obtains in the thorax, it only affects the circulation in the veins more immediately connected with the heart. The great principle, according to Müller, which regulates and maintains the circulation in the veins throughout the body, is the vis a teryo.

We therefore conclude that the diastole of the auricles depends on the same principle, and is produced in a similar manner, as the diastole of the ventricles ; but that, independently of this; the auricles are susceptible of being excited by a stimulus conveyed through the nervous system, causing them to contract with greater vigour, and increase the action of the heart.

\section{REPORTS}

of

\section{MEDICAL AND SURGICAL PRACTICE IN THE HOSPITALS OF GREAT BRITAIN.}

\author{
GUY'S HOSPITAL. \\ CHOREA IN A MAN AGED FORTY: DEATI.
}

(Under the care of Dr. Wilks.)

THE following case, for the report of which we are indebted to Mr. C. H. Spencer, is one of great interest. The symptoms were almost exactly those with which we are familiar in cases of chorea at an earlier period of life ; but such symptoms are very unusual in adult men, and it is perhaps still more remarkable that they should have terminated fatally.

Samuel N., aged 40, gun-maker, Spitalfields, was admitted on September 18 th, 1867 , suffering most severely from an involuntary action of the voluntary muscles. The disease was diagnosed as chorea. The history of the case from its commencement ran as follows. Up to within two years and three months ago, the patient had enjoyed the best of health, and he had been perfectly free from any organic disease. The history stated that the patient was walking down Sackville Street, Spitalfields, one evening about 9.3O P.M., when a man suddenly sprang at him from a recess, and seized him, with intent to garotte him. He, however, escaped from the hands of his would-be captor, and reached his home free from any bodily harm, but in a very excited state of mind. The symptoms of chorea appeared early in the following morning, when he was in the act of playing the violin. He first perceived involuntary movements of his hands and head, and subsequently of his left leg. During the first week of the disease, he placed himself under the care of a medical practitioner in the neighbourhood, and remained under his treatment for three months, but without deriving any benefit. $\mathrm{He}$ remained at home for a further period of three months, during which time the disease gradually increased in severity, affecting all the voluntary muscles. From this time he became unable to feed himself, and could no longer take much solid food, but existed chiefly on a liquid diet.

He now became an in-patient at the Epileptic Hospital, under the care of Dr. Sieveking. The treatment here consisted in galvanism down the spine; but from this he derived no benefit. Sulphate of zinc was then given, and administered for a considerable time; it was thought to have to some extent relieved him. He also took a grain of morphia at night. He continued under the above treatment for five months, after which time, in January, he returned home to Spitalfields, where he remained until September 18th, when he became an inpatient at Guy's Hospital. During the time which had elapsed between his discharge from the Epileptic Hospital and his admission into Guy's, he was galvanised by some private individual, who also attempted to quiet the nervous force by Mesmeric influence. This latter treatment had the effect of quieting him for a short time at intervals. $\mathrm{He}$, however, continued to grow worse daily up to within six or seven weeks of his admission into Guy's. At that time, the disease somewhat abated, so that he could even steady his hands sufficiently to thread a needle. This state of apparent quiescence continued for three or four weeks, when the violent movements again commenced, and continued to increase until the time of his admission. The whole frame, from head to foot, was then in a condition of violent and continued agitation.
He had often bitten his tongue, but not so severely as to cause hæmor. rhage. Since the time of his admission into the Epileptic Hospital, he had been in the habit of taking every night a grain of morphia, without which he got no sleep at all. Its influence only extended over a few hours; and, when once he was aroused, its effects ceased altogether, and the contortions again commenced.

On his admission into Guy's, the condition of the patient was such as to evoke feelings of pity from all those who beheld him. His body was continually undergoing the most violent contortions, the head being tossed to and fro by the involuntary action of the muscles. In some parts of the body, the cuticle was abraded by the violence of the movements. To preserve his tongue from being bitten, he had constantly to keep some portion of bedclothes (such as the edge of the pillow or sheeting) in his mouth. By very great muscular effort, and by clutching some firm object, he could steady himself so as to remain motionless for a minute whilst performing the act of deglutition, which was itself somewhat difficuit to him. He suffered greatly from thirst. A peculiar phenomenon of the disease, which the patient particularly made known and showed to Dr. Wilks, was that he could not lie upon his right side. Whenever he attempted to do so, he was thrown violently to the side on which he lay. The urine was of specific gravity ror9; it contained no albumen. There was no history of syphilis or rheumatism.

Sept. Igth. He was much in the same condition, but perhaps not quite so violent. He was ordered to have a grain of morphia at night, and one-twelfth of a grain of strychnia three times a day.

Sept. 2oth. He did not sleep so well during the night; he was still violent, but rather exhausted from fatigue. He cried out loudly. He was very much parched. He was ordered to be kept under the influence of chloroform, which was commenced at 4 P.M. The smallest quantity rendered him quiet, but he required it to be constantly admi. nistered.

Sept. 2Ist. With the chloroform and morphia, he passed a good night, having slept soundly, there being a brief interval only at 4 A.M. He then took nourishment; and forcible movements came on, but these were easily subdued by chloroform, which was continually administered, allowing only brief intervals, during which the patient took nourish. ment, consisting of beef-tea, eggs, milk, etc.

Sept. 22nd, 2 P.M. Twice within the last two hours, he had had hiccough. -9 P.M. He was still violent, but easily subdued. The effects of the chloroform soon passed off, and then he was more violent than ever. The greatest difficulty was experienced in giving him food. Morphia was repeated as before. - IO P. M. He had recovered from the influence of chloroform. He was very violent, requiring three persons to restrain his movements, in order to prevent injury.

Sept. 23rd, 7 A.M. He passed a good night, having slept well since I I P. M., with an interval of a few minutes at $2 \mathrm{~A}$. M. Nourishment was then administered, and he fell asleep without the aid of chloroform.I P.M. He had been tranquil since the morning. His skin was perspiring. His pulse had been good throughout.

Sept. 24th. He continued in the same condition, easily quieted, but most violent when consciousness returned. The urine was retained; there was some difficulty in passing an instrument.

Sept. 25th, 2 A. M. The morphia appeared to have no further effect on the nervous system in procuring sleep. He required the administration of chloroform every half-hour, in considerably increased quantity. $-9 \mathrm{A.M}$. He was very violent, requiring six persons in the ward to hold him in bed. The breathing had become altered within the last twentyfour hours. He frequently remained half a minute without breathing, and then made a very deep inspiration. The pupils were more dilated than before. His answer was "yes" to every question put to him. When consciousness returned, he always asked for the "ether", as he termed the chloroform.

Sept. 26th. He took the chloroform for the last time at 6 A.M. He then sank into a comatose condition, with convulsions at intervals. $\mathrm{He}$ never recovered his senses, but died at I I A. Mr.

Autopsy, made by Dr. Moxon, three hours after death. There were no signs of decomposition. Rigor mortis was very strong. The bones of the skull were thick; the arteries were healthy. The brain was shrunken; the convolutions were small. The lower lobe of the right lung contrasted with the other lobes; it was heavy, bulky, engorged with blood and water, and easier to break down than the remainder of the organ. The right bronchus was deep red; its epithelial layer was loose and thick, forming a layer almost like that seen in diphtheria on the surface of the membrane. The heart weighed nine ounces; it was remarkably contracted. The valves were healthy. The large intestine was considerably loaded with freces. The liver was very fatty. 